This randomized, placebo-controlled, doubleblind study included 457 men aged at least 50 years with an International Prostate Symptom Score (IPSS) of 8 or more. Patients were randomly allocated to receive oral silodosin ( $4 \mathrm{mg}$ twice a day), tamsulosin (0.2 mg daily), or placebo (twice a day) for 12 weeks, following a 7-day wash-out period and a 7-day observation period. The primary endpoint was the change in IPSS from baseline.

Patients received either silodosin $(n=176)$, tamsulosin $(n=192)$, or placebo $(n=89)$. The total IPSS decreased from baseline by a mean of $8.3,6.8$, and 5.3 in the silodosin, tamsulosin, and placebo groups, respectively. Within a week of starting treatment, there were significantly greater decreases in IPSS with silodosin than with placebo. The incidence rates of drugrelated adverse events were $69.7 \%, 47.4 \%$, and $36.4 \%$ in the silodosin, tamsulosin, and placebo groups, respectively.

From the results of this study, the authors conclude that silodosin is useful for the treatment of LUTS associated with BPH.

Original article Kawabe K et al. (2006) Silodosin, a new $\alpha_{1 \mathrm{~A}}$-adrenoceptor-selective antagonist for treating benign prostatic hyperplasia: results of a phase III randomized, placebo-controlled, double-blind study in Japanese men. BJU Int 98: 1019-1024

\section{Feedback transurethral microwave thermotherapy treats BPH effectively}

Transurethral microwave thermotherapy can be used to treat benign prostatic hyperplasia (BPH). The CoreTherm ${ }^{\circledR}$ device (ProstaLund Operations AB, Lund, Sweden) monitors intraprostatic temperature and adjusts the microwave power accordingly to deliver customized thermotherapy, which is necessary because intraprostatic blood flow varies and can act as a heat sink. Schelin et al. compared the efficacy and safety of CoreTherm ${ }^{\circledR}$ thermotherapy to that of surgery.

Schelin and colleagues' prospective, controlled, multicenter study enrolled 120 patients (aged $\geq 45$ years) with symptomatic BPH and persistent urinary retention that required catheterization. The patients were randomly assigned to receive CoreTherm ${ }^{\circledR}$ thermotherapy $(n=61)$ or surgery (49 patients underwent transurethral resection of the prostate and 10 underwent enucleation), and were followed up at 3 and 6 months.

In total, 54 thermotherapy-treated men and 52 surgically treated men completed the study. At 3 months, $79 \%$ of thermotherapy-treated patients and $86 \%$ of surgically treated patients had their catheter permanently removed; they all remained catheter-free at 6 months. The mean catheterization time was 34 days for thermotherapy-treated patients and 5 days for surgically treated patients. Serious adverse effects were reported in more surgically treated than thermotherapy-treated men $(8.5 \%$ versus $1.6 \%$, respectively). The most common adverse effect was urinary tract infection (33\% of thermotherapy-treated men and $22 \%$ of transurethrally resected men).

The authors conclude that CoreTherm ${ }^{\circledR}$ thermotherapy is an effective alternative to surgery for $\mathrm{BPH}$ and provides a treatment option for elderly, fragile men who cannot undergo surgery.

Original article Schelin S et al. (2006) Feedback microwave thermotherapy versus TURP/prostate enucleation surgery in patients with benign prostatic hyperplasia and persistent urinary retention: a prospective, randomized, controlled, multicenter study. Urology 68: 795-799

\section{Long-term $\alpha$-adrenergic- antagonist treatment alleviates symptoms of CP/CPPS}

Chronic prostatitis and chronic pelvic pain syndrome (CP/CPPS) are difficult to treat, and the efficacy of empiric treatments such as $\alpha$-adrenergic antagonists remains unproven. Yang et al. performed a meta-analysis of nine studies in which a total of 734 patients with CP/CPPS (NIH category III prostatitis) were randomly allocated to receive either an $\alpha$-adrenergic antagonist or placebo. Treatment duration varied from 4 weeks to 6 months.

Long-term ( $>3$ months) use of $\alpha$-adrenergic antagonists significantly decreased symptoms in patients with CP/CPPS, compared with patients who received placebo, mainly owing to a decrease in severity of urinary symptoms (standardized mean difference $-1.60,95 \% \mathrm{Cl}$ -2.24 to -0.96$)$. The authors had hypothesized that $\alpha$-adrenergic antagonists relax the urinary bladder neck and prostatic urethra, which prevents reflux of urine and improves detrusor stability. However, their analysis showed no 